
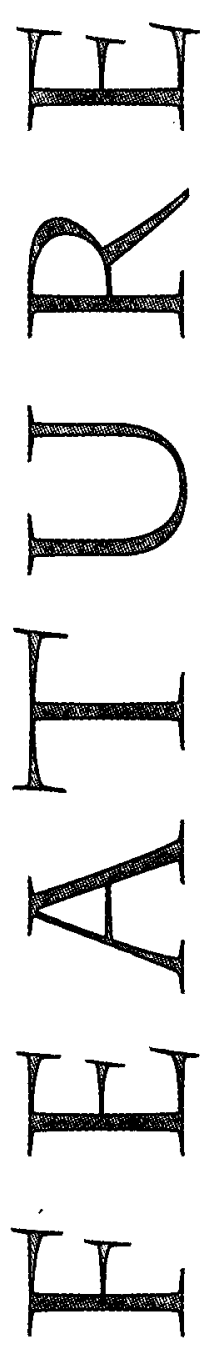

\section{Research in Managed Care: Outcomes Research in Disease Prevention and Management}

The relationship between outcomes research - whether clinical, economic, humanistic, or a combination-and disease management is explored in this feature. Learn why it's important to implement the concepts behind these termsand not view them as simply the latest buzzwords.

I

f buzzwords are terms that are overused and ill-defined, two leading candidates in the health care field would be outcomes research and disease management. This article will attempt to define these two concepts; discuss how outcomes research provides a critical need in conducting disease management; give an example of an outcomes research plan for evaluating a disease-management program; and provide some thoughts regarding the global issues concerning disease management.

DISEASE PREVENTION AND MANAGEMENT: DOING THE

\section{TOM HUGHES}

- TOM HUGHES, R.PH., PH.D., is Director of Outcomes Research, PCS Health Systems, and Adjunct Professor, Center for Pharmaceutical Economics, College of Pharmacy, University of Arizona, Tucson. RIGHT THING

At PCS Health Systems, the professional staff prefers the term "disease prevention and management" because it is more comprehensive than the term 


\section{EXAMPLE OF OUTCOMES RESEARCH PLAN}

\section{Step 1: Evaluate Current Delivery of Care}

Identify the current delivery of care for a defined diabetic population, from a quantitative data perspective. This step evaluates the care that diabetic patients are receiving in a *health care organization, using a variety of data sources that may be available (e.g., pharmacy and medical claims). In general, one wants to describe: (1) patient demographics, (2) the clinical processes patients are receiving, and (3) the clinical outcomes the patients are experiencing.

Patient demographics are important in understanding a diabetic population. Are they young (e.g., Type I diabetics at a higher risk for diabetic ketoacidosis hospitalizations), are they primarily middle age Type II patients who may be candidates for diet and exercise interventions, or are they elderly Type II patients beginning to experience the long-term effects of diabetes?

Clinical processes are important in understanding whether the care being provided is optimal relative to some standard of care, such as the American Diabetes Association guidelines. In the rush to embrace outcomes, clinicians sometimes forget the importance of process. The result of poor clinical process (e.g., no HbAlc tests for a diabetic patient) will be poor clinical outcomes. Clinical outcomes are still necessary to measure, as they will document the outcomes associated with the interventions. The clinical outcomes should be selected to evaluate the downstream results of the clinical processes being measured.

While the process of care in a specific disease state can be assessed in a quantitative manner using claims data, a qualitative evaluation of the process of care is also essential in understanding the process of care. This qualitative evaluation can be conducted using a person with clinical expertise to interview providers of care, recipients of care, and payers of care. The providers should include at a minimum a primary care physician, a specialist, and a nurse, all of whom deal with patients with the selected disease state and are members of the health care plan. In addition, a sample of the patients should be interviewed. These qualitative interviews can provide insight into why certain results exist within a health care organization. For example, a clinical process deficiency may occur in a health care organization that does not provide reimbursement for a specific clinical procedure. The results from the qualitative interview should be integrated with the results from the quantitative data analysis to evaluate how care is being provided to a defined population in a given health care plan.

\section{Step 2: Specify Interventions}

In synthesizing the baseline evaluation, deficiencies within the plan may be identified. The next step is to specify interventions to address these deficiencies. Specifying the interventions is not an easy step, but, as this is not the focus of this article, it will not be addressed here.

\section{Step 3: Outcomes Research Design}

Based on the interventions that have been identified for implementation in the health care organization, the outcomes measurement plan is developed. The plan should follow the clinical decisions made regarding the interventions to be implemented in the health care organization. This process is initiated by working with the clinicians who are designing the interventions. To specify the outcomes measurement plan, one needs to have a clear understanding of what the clinician expects from the interventions.

An outcomes measurement plan can then specify the clinical, economic, and humanistic outcomes to be measured, how often measurement will occur, and what data are neces- disease management. This is not an attempt to coin a new buzzword, but more specifically to state clearly the ultimate locus of efforts. The concern is with the entire spectrum of health care, and disease prevention and management recognizes the fact that, when possible, disease-prevention efforts should be included in how the care plan is provided, as opposed to waiting to intervene after a patient has developed the disease.

For this article, I will adapt an often-quoted pharmacy objective to define disease prevention and management. A central task of pharmacists is to provide the right patient with the right drug at the right time. The essence of disease prevention and management can be defined as providing the right information to the right decision-maker at the right time.

The right information includes the type of data and how it is delivered. Any information necessary for the patient, the patient's family, or the health care provider to prevent or manage a disease optimally is included. In addition to the type of information, choosing the delivery system for this information is critical. The information must be "packaged" to achieve the desired im- pact on the decision-maker who receives it. For example, patient education information must be in a language and at a reading comprehension level that the target group of patients can understand. The information given to providers must present a clear and concise message to elicit the desired behaviors from the provider.

The decision-maker can be the health care provider and might include physicians, pharmacists, nurses, patients, or patients' families. For a pharmacist dispensing an antibiotic, the

Continued on page 216 
sary to conduct the measurement. Table 3 contains an example of an outcomes measurement plan for diabetes.

\section{Step 4: Data Requirements}

The data necessary to conduct the analyses can then be stated. This is an essential step, conducted in parallel with the measurement plan. If an outcome is specified in the measurement plan, then the data necessary for measuring it must be available.

The necessary data may be difficult to obtain. For example, obtaining information about patients' medical history may be helpful in targeting interventions, but this information is not easy to access. One would either need to review the medical records or conduct a patient self-report. While data such as patient medical history are accessible, the cost of obtaining these data is relatively high, compared with medical and pharmacy claims. The cost of accessing data must be balanced with the value it provides. Collecting data from chart review may be reasonable, if the expected outcomes of the interventions cannot be reliably measured from claims data. In other cases, information from claims data may be sufficient.

\section{DISEASE-MANAGEMENT BIBLIOGRAPHY}

Anders G. Drug makers help manage patient care. Wall Street J 1995 (May 17): B1, B7.

Armstrong EP, Langley PC. Disease management programs.

Am J Health-Syst Pharm 1996; 53: 53-8.

Bernard S. Disease management: a pharmaceutical company perspective. Pharm Exec 1995; 15(3): 48-60.

Broshy E, Matheson D, Hanson M. Want to curb health costs? Manage the disease, not each cost component. Med Market Media 1993: 76-84

Castagnolia WG. Is disease management good therapy for an ailing industry? Med Market Media 1995 (Jan): 46-53.
Cohen KR, Naughton DH. Patient-centered care: a revolution in medicine. Med Interface 1995; 8(6): 66-73.

Hadsall RS, Sargent LJ. Disease state management. J Managed Care Pharm 1995; 1: 127-32.

Koberstein W. Real reform: toward a true partnership. Pharm Exec 1995; 15(4): 38-60, 74-6.

Koberstein W. Real reform: where disease management is taking the industry. Pharm Exec 1995; 15(4): 36-7.

Landis NT. Disease management entries multiply, diversify.

Am J Health-Syst Pharm 1995; 52: 1268-72.

Lumsdon K. Disease management: the heat and headaches over retooling patient care create hard labor. Hosp Health Networks 1995; 69(7): 34-42.

Madel R, Peck A, DiPalma K. Real reform: other voices.

Pharm Exec 1995; 15(4): 62-72.

Mandelker J. Managing chronic disease. Bus Health 1994; 12(11): 45, 49-50.

Marro EP. Is disease state management what customers really want? Med Interface 1995; 8(11): 144-6.

Moench E. What does DM mean to patients? Pharm Exec 1995; 15(5): 52-64

Muirhead G. Disease management: threat or opportunity for pharmacy? Drug Top 1995; 139(15): 50-9.

Terry K. Disease management: at these companies, the future is now. Bus Health 1995; 13(4): 73-6.

Terry K. Disease management: continuous health-care improvement. Bus Health 1.995; 13(4): 64-72.

Trice SB. DUE: moving toward disease management. Consult Pharm 1995; 10: 402-20.

Zalta E, Eichner H, Henry M. Implications of disease management in the future of managed care. Med Interface 1994 :

7(12): 66-78.

Zitter M. Disease management: a new approach to health care. Med Interface 1994; 7(8): 70-6.

Zitter M. Disease management: a systems approach to managing health outcomes. Med Interface 1995; 8(1): 70-90. right information may be inquiring about a patient's drug allergy history. A physician can use the right information to recognize the need for an asthma patient to have an inhaled corticosteroid added to a treatment regimen. For a patient with diabetes, the right information may be instructions on how to check blood glucose.

The right time means making sure the decision-maker has the necessary information when a decision needs to be made. This requires integrating the inputs from a variety of data sources to deliver a comprehensive package of information in a nonintimidating or non- burdensome manner to the decisionmaker. If accessing or using these packages-even if it resides in an integrated form-takes more effort than the perceived value, the decision-maker will not do so.

Some would say disease prevention and management is nothing new. It is just providing appropriate clinical care to patients, something that has been done for many years. While this has been the implicit goal of health care, disease prevention and management has an ally in the 1990s that was not available to health care providers in prior decades: information technology.
Information technology will play a central role in making disease prevention and management successful. Information technology allows timely collection, analysis, and dissemination of data. This can be demonstrated via something as "simple" as the advent of a patient drug allergy clinical software system for checking individual patient allergy information when filling a prescription. A more sophisticated example would be a physician being able to access in real-time a patient's complete medical history, via an electronic med-

Continued on page 221 
Table 1. Diabetic Outcomes Measurement Plan

\begin{tabular}{|c|c|c|c|c|c|}
\hline & Baseline & Three months & Six months & Nine months & 12 months \\
\hline \multicolumn{6}{|l|}{ Clinical } \\
\hline \multicolumn{6}{|l|}{ Mean number of HbAlc tests } \\
\hline per patient & $\checkmark$ & $\checkmark$ & $\checkmark$ & $\checkmark$ & $\checkmark$ \\
\hline Mean HbAlc level & $\checkmark$ & $\checkmark$ & $\checkmark$ & $\checkmark$ & $\checkmark$ \\
\hline Mean patient compliance with & & & & & \\
\hline oral hypoglycemics & $\checkmark$ & & & & $\checkmark$ \\
\hline $\begin{array}{l}\text { Mean number of hospitalizations } \\
\text { per } 1,000 \text { covered lives }\end{array}$ & $\checkmark$ & & & & $\checkmark$ \\
\hline Mean hospital length of stay & $\checkmark$ & & & & $\checkmark$ \\
\hline $\begin{array}{l}\text { Mean number of emergency } \\
\text { room admissions }\end{array}$ & $\checkmark$ & & & & $\checkmark$ \\
\hline Humanistic & & & & & \\
\hline Patient health status & $\checkmark$ & $\checkmark$ & $\checkmark$ & $\checkmark$ & $\checkmark$ \\
\hline Patient satisfaction & $\gamma^{a}$ & & & & \\
\hline $\begin{array}{l}\text { Physician satisfaction } \\
\text { Economic }\end{array}$ & $\sigma^{a}$ & & & & \\
\hline Direct economic costs & $\checkmark$ & $\checkmark$ & $\checkmark$ & $\checkmark$ & $\checkmark$ \\
\hline Indirect economic costs & $\checkmark$ & $\checkmark$ & $\checkmark$ & $\checkmark$ & $\checkmark$ \\
\hline
\end{tabular}

a Assesses satisfaction with educational material.

Figure 1. Outcomes Categories

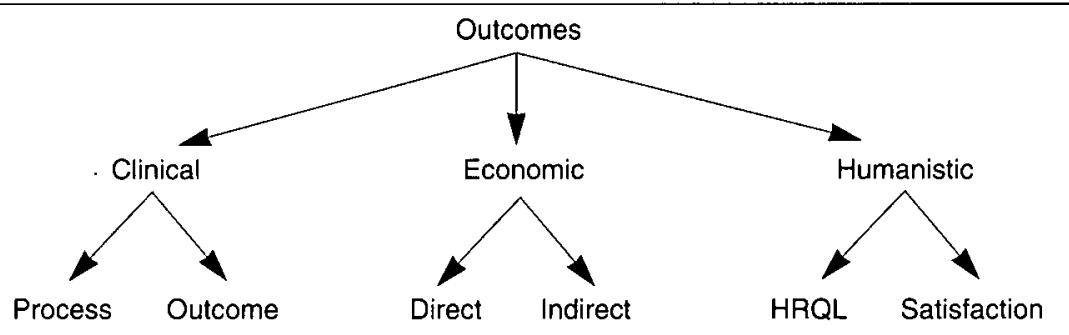

ical record, while assessing the effects of therapy on resulting laboratory, diagnostic, and clinical results for the treatment of a disease episode.

For more information on disease prevention and management, see the bibliography on disease prevention and management (page 216). This list was created at the Center for Pharmaceutical Economics at The University of Arizona.

\section{OUTCOMES RESEARCH}

Research in health care can be viewed as collecting data from a variety of sources (e.g., pharmacy claims, medical claims, laboratory results, physician chart notes) and transforming them into information that answers important questions. Outcomes can be categorized as clinical, economic, or humanistic (Table 1). A case can be made for a causal relationship between the three outcomes, with the clinical outcomes driving downstream economic and humanistic outcomes.

Clinical outcomes can be delineated into process and outcome measures. Clinical process measures can be found in treatment guidelines or guidelines developed by NIH consensus panels. Using diabetes as an example, a clinical process measure would be the percentage of diabetic patients receiving an annual retinal eye examination. Clinical outcome measures evaluate health care use that results from either optimal or suboptimal adherence to clinical processes. Again, using diabetes as an example, poor process will lead to undesirable health care use (e.g., hospitalization for diabetic ketoacidosis).

Economic outcomes can be delineated into direct economic costs and indirect economic costs. Direct economic costs are what health care practitioners traditionally think of as costs in the health care system, such as inpatient care, outpatient care, pharmacy, and laboratory tests. Indirect economic costs are represented by productivity losses. Three examples of indirect costs are premature mortality, lost work days, and decreased productivity while at work.

Humanistic outcomes can be delineated into health-related quality of life and satisfaction. Health-related quality of life, also referred to as functional status or health status, is patient derived, via a questionnaire or interview. Satisfaction is a broad measure, and either provider or patient satisfaction can be assessed. When considering satisfaction, a question to ask is, satisfaction with what? For example, satisfaction can be assessed about an educational intervention a patient received, how long it takes a patient to see a health care provider, or friendliness of the staff.

The objective of outcomes research and its role in disease management is to affect disease states longitudinally rather than during a single episode. Often outcomes research is used in a study that evaluates the outcomes associated with an intervention for a specific study peri- 
od (e.g., a one-year evaluation of an intervention in asthma). Outcomes research associated with disease management is an ongoing initiative; it is continually evaluating the clinical, economic, and humanistic outcomes associated with the interventions. This ongoing evaluation allows monitoring of the patient interventions. If the population changes, resulting in the need for a modification of an intervention, the ongoing outcomes research initiative will identify this need. If an intervention ceases to be effective in a stable population, the ongoing research initiative will again identify this need and alert the individuals responsible for the design and implementation of the interventions of the need for modification.

\section{INTERACTION BETWEEN DISEASE PREVENTION AND MANAGEMENT AND OUTCOMES RESEARCH}

I have briefly reviewed the definitions of disease prevention and management and outcomes research. How do these two concepts interact? As a starting point, disease prevention and management programs are conducted for a reason, usually to favorably affect one or more of the outcome categories described above. Outcomes research provides a reliable and valid approach for documenting the outcomes realized from disease prevention and management programs. If the outcomes of interest cannot be measured, then it is impossible to show that the disease prevention and management program resulted in any differences. In addition, the outcomes themselves are important pieces of information when incorporat- ed into delivering the right information to the right decision-maker at the right time. Additionally, they provide feedback for future health care decisions.

Shown in the sidebar on page 214 is a practical example using diabetes mellitus and illustrating some of the issues encountered when creating an outcomes research plan for disease management.

\section{MINOR REVELATIONS: PERSONAL REFLECTIONS}

After having two years of experience in disease prevention and management, I have a few thoughts regarding the field.

A mantra within the disease prevention and management world is that if quality improves, outcomes will improve. At a certain level I agree with this statement, as long as no one is too particular regarding the outcomes or the disease state. I think this statement can be approached from two perspectives, the clinical and the financial perspective.

From a clinical perspective, there is a tremendous need to increase the quality of care we are providing, especially with regard to chronic diseases. I believe that as you increase the quality of care in general, clinical outcomes will improve. Again, process leads to outcomes; in an environment where everyone is doing the right stuff, good things will happen.

From a financial perspective, however, it becomes a little sticky. First of all, improving the quality of care incurs some direct economic costs. If a net direct economic savings is expected, the savings must be greater than the intervention costs-no surprises so far. Not all disease states provide equal "oppor- tunity" to realize net direct economic savings in the short term by conducting disease prevention and management.

One issue is that current financial evaluations often are only looking at direct economic dollars. It is not uncommon for indirect costs of a disease to represent a substantial portion of the illness. The difficulty is that researchers have not done a great job documenting indirect costs in a reliable and valid manner, as the data for this initiative are largely not captured through formal means.

My second thought focuses on the premise of "low hanging fruit"-the idea that disease prevention and management is actually fairly easy to do. This is a fallacy; if it were easy to do, health care organizations would have picked this fruit by now. Changing the care process associated with a disease state is not an easy task, but it is where the opportunities exist. Witness asthma: in 1991, the NIH consensus panel report determined that asthma was an inflammatory process, requiring an inhaled corticosteroid as a critical component of a patient's therapy. Five years later, medicine and health care are still far from having this relatively simple initiative used in the majority of asthma patients.

\section{CONCLUSION}

Disease management is a buzzword. The ability to understand the process of care, create interventions, and implement and manage interventions is easier to talk about than to do. However, the ability to provide the right information to the right decision-maker at the right time holds great potential for improving the provision of health care to the most important person in the system, the patient. 